\title{
Wing morphology in migratory North American monarchs: characterizing sources of variation and understanding changes through time
}

https://doi.org/10.1515/ami-2018-0003

Received June 15, 2018; accepted September 20, 2018

\begin{abstract}
Monarch butterfly wing morphology varies substantially throughout their global range, both between resident and migratory populations and also within the migratory North American population. Here, we use a dataset comprising more than 1800 North American individuals collected between 1878-2017 to characterize the factors shaping continent-wide patterns of wing morphological variation. North American overwintering butterflies have forewings that are approximately $4.4 \%$ larger than those collected in summer breeding areas. Monarchs overwintering in Mexico have forewings that are approximately $1.8 \%$ larger than monarchs overwintering in California, conducive to the idea that migration distance is positively correlated with wing area. We find evidence for a latitudinal cline within North America, such that butterflies collected at higher latitudes have significantly larger and more elongated forewings. We also find a significant increase of approximately $4.9 \%$ in forewing area between 1878-2017, but no difference through time in wing elongation. This result is corroborated by a reanalysis of a recently published dataset of more than 600 butterflies from Mexican overwintering sites. We discuss possible reasons for this increase in wing size through time, including northward shifts in the monarch's breeding range and changes in relative abundance of milkweed hosts, and present experimental data addressing the influence of larval host plant on adult wing morphology. Our analysis suggests that (1) migration is indeed an important selective force for monarch wing
\end{abstract}

\footnotetext{
*Corresponding author: Micah G. Freedman, Department of Evolution and Ecology, University of California, Davis, E-mail: mfreedman@ucdavis.edu

Micah G. Freedman, Center for Population Biology, University of California, Davis

Hugh Dingle, Department of Entomology and Nematology, University of California, Davis
}

morphology; (2) wing size has increased through time in North America; (3) factors such as host plant identity must be considered to fully understand monarch wing morphological variation.

Keywords: monarch butterfly, migration, morphology, evolution, museum collections

\section{Introduction}

Migration has evolved across the tree of life as a way for organisms to exploit ephemeral or only seasonally available resources, often over enormous spatial scales. In organisms that migrate long distances, traits conducive to efficient movement are imperative. This can entail certain behavioral and physiological adaptations, such as periods of reproductive dormancy and lipid accumulation prior to or during migration [1,2], as well as morphological features that aid in long-distance movements. In the latter case, selection has favored certain body shapes in migratory fishes [3,4], wing loading patterns in migratory birds [5,6], and wing sizes and shapes in migratory insects $[7,8]$.

Perhaps the best-known migratory insect is the monarch butterfly, whose seasonal migration spans thousands of kilometers across the North American continent. Past research has shown that migratory and non-migratory populations of monarch butterfly have highly divergent wing morphologies, with migrants typically having larger, more elongated wings than nonmigrants [8-11]. This pattern has been interpreted as natural selection operating on a tradeoff between longdistance flight in migrants and short-distance flights in summer breeding and non-migratory populations. Large, elongated wings are thought to aid in gliding flight and facilitate long-distance movements in migratory monarchs [8], whereas smaller, rounder wings are associated with maneuverability that may be more important for summer- 
breeding or non-migratory monarchs [10].

In addition to wing morphological differences between residents and migrants, studies have shown correlations between timing of migration, wing coloration, and wing size in North American migrants [12-15]. Likewise, two recently published studies using stable isotope data have shown that butterflies that migrate longer distances to overwintering sites have larger forewings [16,17]. An earlier study by Altizer and Davis (2010) also found differences in forewing area between eastern and western North American monarchs, which they attribute to differences in migration distance [8]. These results have been interpreted as migration acting as a selective episode on wing morphology, such that the largest and most capable long-distance migrants disproportionately reach overwintering grounds.

A number of factors areclearly important determinants of wing morphology in monarchs: for example, males are consistently larger than females, and as noted above, migration distance to overwintering sites seems to be positively correlated with forewing area. While these findings provide a useful starting point for understanding wing morphological variation among North American migrants, a number of other potentially important factors have yet to be fully considered. For example, the extent to which wing morphology is phenotypically plastic is not well-studied, and few studies have considered how factors such as larval photoperiod and host plant identity might impact adult wing morphology (but see refs. 18,19).

One approach to understanding this variation is by conducting experimental manipulations to test specific hypotheses about how certain factors influence monarch wing morphology. Another approach is to take advantage of the extensive museum collections of North American monarchs and their associated metadata to build a comprehensive model that allows for testing multiple hypotheses about wing morphological variation. While the latter approach has the obvious shortcoming of integrating over many sources of uncertainty, an advantage is that it enables the evaluation of certain hypotheses that could not otherwise be tested, such as possible changes through time, both within and across years.

In this study, we combine data from museum specimens and contemporary collections of monarch butterflies to create a database of more than 1800 North American individuals. We then use linear mixed effects models to understand sources of variation in monarch forewing morphology. Specifically, we evaluate the effects of butterfly sex, overwintering status, latitude of collection, year of collection, photoperiod at time of collection, and membership in the eastern or western migratory population as predictors of forewing size and shape. We then compare a subset of our data to a recently published dataset of Mexican overwintering monarchs [17] to compare trends through time. Finally, we conduct a common garden rearing experiment using a split brood design to understand the impacts of larval host plant on adult wing morphological variation.

\section{Methods}

\subsection{Data Collection}

Specimens included in this study are primarily from museum collections, with some butterflies coming from previously published papers and others from contemporary collections of North American individuals (Table 1). For museum specimens, butterflies were photographed using either a Panasonic Lumix FZ80 (Panasonic Corp., Osaka, Japan) or Nikon D7100 (Nikon Corp., Tokyo, Japan) camera mounted on a camera stand or tripod. All images included a scale bar, and butterflies were placed so that forewings were in the same horizontal plane as this scale bar (Figure 1). For some individuals, wings were not spread evenly during pinning; in these cases, we angled the butterfly such that only one pair of wings was planar with the scale bar. Butterflies were photographed individually and were positioned to be at the center of the image to minimize possible effects of radial distortion. For contemporary butterfly collections, wings were clipped at the attachment point to the thorax and imaged using a flatbed scanner (Canon LiDE 120, Canon U.S.A., Inc., Melville, NY) with a scale bar. In total, we included 1804 North American butterflies from 16 museum and private collections (Figure 2, Table 1).

All images were measured using the image processing software ImageJ [20]. Briefly, images were scale calibrated, and then measurements were taken for both left and right forewings. We measured forewing length and width in the same way as previously published studies $[8,16,17]$. We manually defined the outline of monarch forewings by tracing a thin white line around the forewing margin in areas where forewing and hindwing overlapped. We then converted photos into 8-bit black/white images and used the wand tool to select the outline of each forewing. Perimeter was measured either by fitting a cubic spline to the edge of this shape using the 'Spline Fit' feature, or by using the 'Interpolate' feature and selecting an interval of 25-40 pixels depending on the pixel density of the image; in both cases, this was done to minimize noise associated with the delineation of the wing outline. Forewing area 
(a)

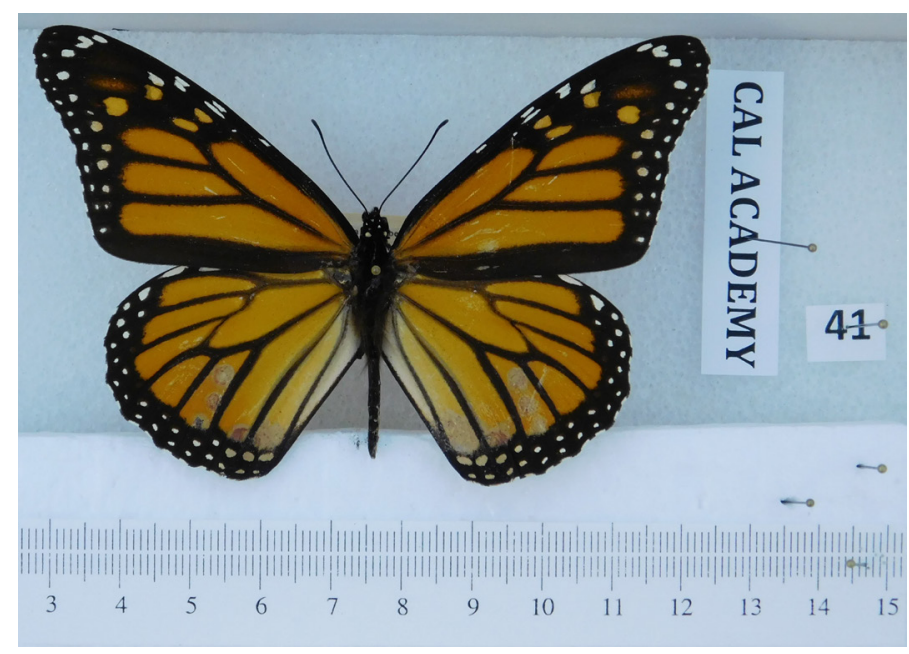

(c)

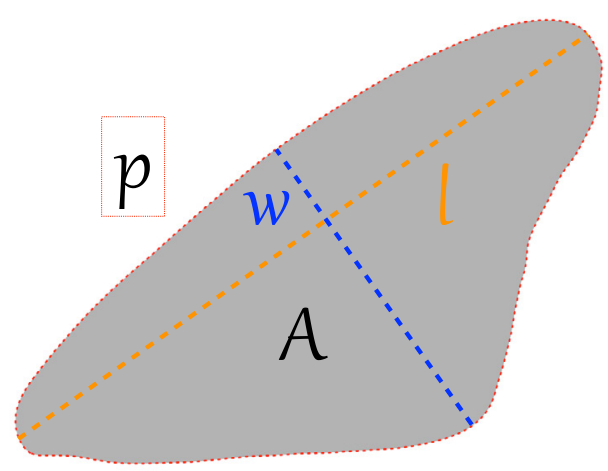

(b)

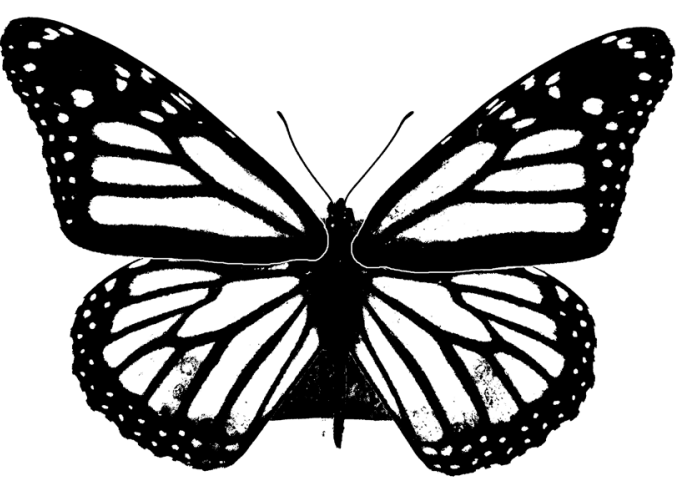

aspect ratio $=\frac{l}{w}$

roundness $=\frac{4 \pi A}{p^{2}}$

Figure 1. Example of image used for data generation. (a). Unaltered image, including scale bar, of a female monarch in the California Academy of Sciences collection. (b). 8-bit black and white image showing delineation of forewing outline. (c). Left forewing and the measurements taken from it. The red outline of the wing corresponds to a spline with 435 discrete points and was used to generate a smoothed estimate of wing perimeter. Length $(\mathrm{l})$, width $(\mathrm{w})$, perimeter $(\mathrm{p})$, and area $(\mathrm{A})$ were measured for both left and right forewings, with averages of these values used to generate measurements of aspect ratio and wing roundness for each individual butterfly. Throughout the text of this manuscript, we use roundness as our descriptor for forewing shape and use this term interchangeably with forewing elongation (greater forewing elongation corresponds to lower roundness).

was calculated as the area within this smoothed outline. In some cases where monarch forewings had minor damage that caused discontinuities in the outline, we manually corrected the outline of the wing. In cases where damage was more severe and the wing outline would have required more extensive interpolation, we omitted wings from analysis. Processing of scanned wings was performed in the same way as described for photographs. In most cases, both left and right forewings were measured for length, width, perimeter, and area. These values were used to calculate forewing aspect ratio and roundness as described in Altizer and Davis (2010) [8]. Briefly, higher values of roundness correspond to less elongated, more circular wing shapes, and we refer to wing shape from hereon in terms of wing elongation. Left and right wing measurements were then averaged into a single mean value for each butterfly.

For each specimen, we recorded the sex of the butterfly and all relevant locality information as well as the date of collection. This locality information was used to generate the latitude and longitude of collection for each individual using the geocode function in the ggmap package [21]. We did not record the elevation of collection, as this was not recorded for most specimens. Likewise, only a small number of specimens included information on possible larval host plant species. Specimens labeled 


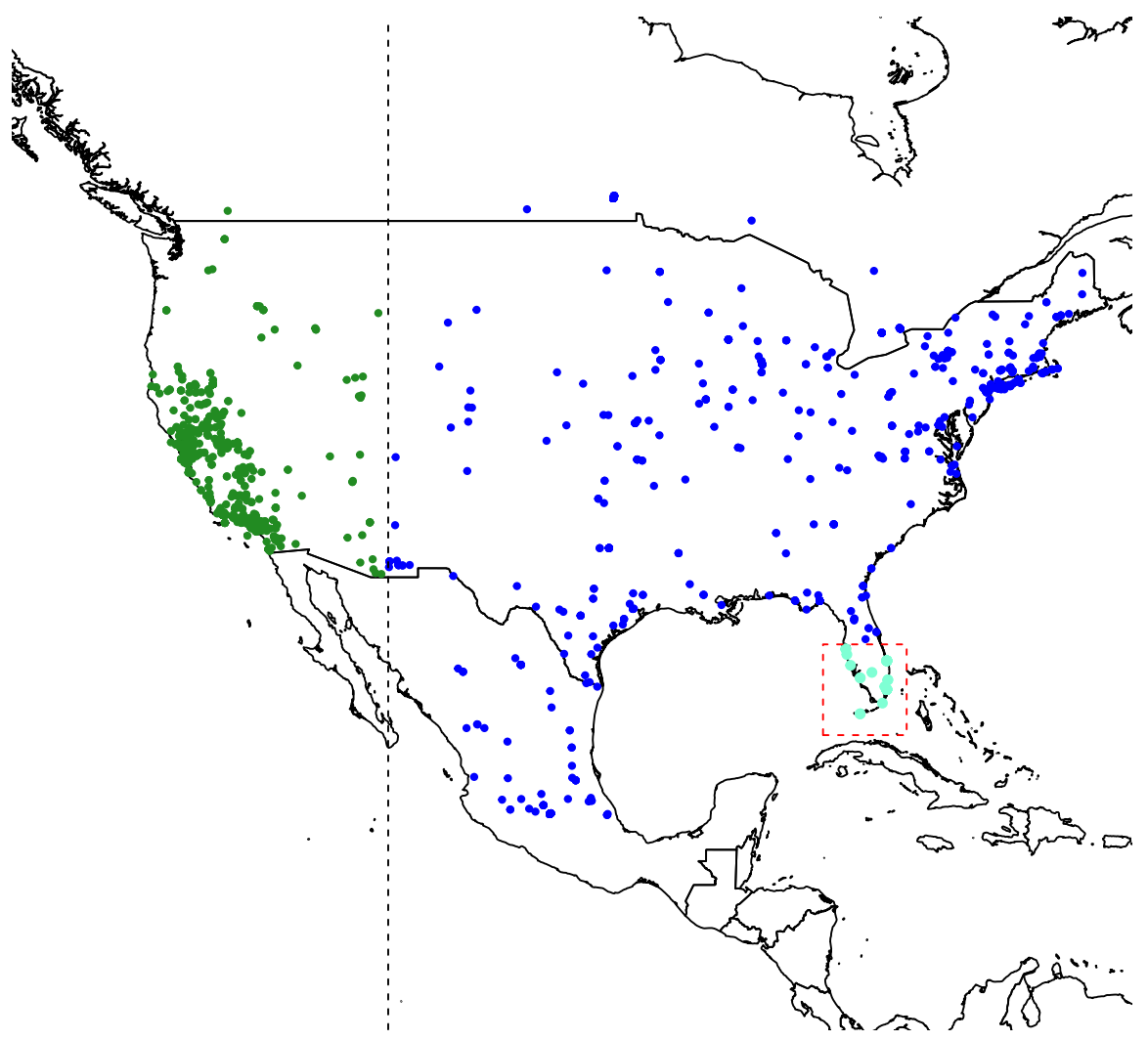

Figure 2. Map of sampling locations for butterflies included in the present study. Eastern North American butterflies are colored in blue, while western butterflies are colored in dark green; the divide between them is shown as a dashed vertical line at $110^{\circ} \mathrm{W}$. Non-migratory monarchs from south Florida are shown in aquamarine and were not included in analyses of continent-wide patterns.

Table 1. Summary of collections used in the current study. Butterflies from Yang et al. (2016) and Li et al. (2016) were the same images used in those studies, but re-measured according to our measurement protocols. Totals do not include butterflies from Florida collected at locations below 28 degrees of latitude $(n=56)$, as these are likely year-round breeding individuals.

\begin{tabular}{lll}
\hline Collection & Count & Collection Year Range \\
\hline American Museum of Natural History & 88 & $1908-1985$ \\
Bishop Museum of Hawaii & 4 & $1953-1953$ \\
Bohart Museum (UC Davis) & 199 & $1918-1999$ \\
California Academy of Sciences & 256 & $1891-2001$ \\
Miscellaneous Contemporary specimens & 19 & $2016-2017$ \\
Cornell University Insect Collection & 154 & $1915-1996$ \\
Mexican individuals - David M. & 66 & $1978-2012$ \\
Essig Museum (UC Berkeley) & 116 & $1919-2002$ \\
Harvard Museum of Comparative Zoology & 73 & $1878-1984$ \\
Los Angeles County Museum of Natural History & 190 & $1902-1996$ \\
Yang et al. (2016), Ecography & 182 & $2011-2012$ \\
Li et al. (2016), Animal Migration & 57 & $2007-2012$ \\
McGuire Center (University of Florida) & 83 & $1928-2012$ \\
Personal Collection: Myron Zalucki & 11 & 2009 \\
Peabody Museum (Yale University) & 19 & $1949-1978$ \\
UC Riverside Insect Collection & 74 & $1917-2004$ \\
Smithsonian & 109 & $1885-1989$ \\
Cumulative total & 1804 & $\mathbf{1 8 7 8 - 2 0 1 7}$ \\
\hline
\end{tabular}


as 'ex-ovum' or 'ex-pupa' were omitted from analysis. For overwintering status, we recorded butterflies as belonging to an overwintering population if they were collected from a known overwintering site between November 1 February 15, or if the specimen label explicitly indicated membership in an overwintering population. Butterflies were recorded as belonging to eastern or western North America based on the longitude of collection, with all individuals from west of $110^{\circ} \mathrm{W}$ treated as western North American (see Figure 2).

For collection dates, we included the year, month, and day of collection when available. When month and day of collection were available, we calculated the Julian date of collection. Using the Julian date of collection, we created an index for photoperiod at the time of collection (hereby daylength index), with the minimum and maximum values at the winter and summer solstices, respectively. We included this term to account for the possibility that larval photoperiod might influence adult wing morphology [19], though we acknowledge that there may be substantial time lags between larval development and date of collection for adults. For a distribution of specimens across Julian dates and a visual representation of the daylength index, see Figure S1.

\subsection{Data Analysis}

All data were analyzed using linear mixed models in the lme4 package [22] in R version 3.4.4 [23]. We used wing area as the response variable for analyses addressing forewing size, as this value was almost perfectly correlated with wing length $\left(R^{2}=0.925\right)$ and width $\left(R^{2}=0.945\right)$ (see Figure S2). For analyses of wing shape, we used roundness as our response variable. All continuous predictor variables were centered and scaled. Since we were primarily interested in variation within North American migrants, we omitted non-migratory butterflies from Florida by excluding Florida specimens collected further south than $28^{\circ} \mathrm{N}$ (see Figure 2).

In all models, we included collection ID as a random intercept to account for possible discrepancies between collections in image generation or scale calibration. We also included a random intercept term for the state of collection (e.g. Alabama vs. Arkansas vs. California, etc.) to partially account for spatial non-independence of sampling in our dataset. For fixed effects, we included butterfly sex, latitude of collection, overwintering status, membership in eastern versus western North America, and daylength index. We also include an interaction term between overwintering status and eastern versus western North America, based on results from previous studies about migration distance acting as a selective filter on wing morphology.

We compared our data to the recently published dataset in Flockhart et al. (2017) [17]. Their dataset comprised 613 overwintering butterflies from a number of Mexican overwintering sites, spanning the years 19742014. Using their online supplementary information, we re-analyzed their data with a simple linear model that evaluated wing area as a function of migration distance (using the distance to centroid measure), sex, and year of collection. For comparison between datasets, we restricted our results to the same time period (1974-2014) and included only non-overwintering individuals $(\mathrm{n}=493)$ to determine whether wing morphology for overwintering and summer-breeding butterflies showed similar patterns over this time period.

\subsection{Host plant experiment}

As part of a different experiment, we evaluated the contribution of larval host plant to adult forewing morphology. Milkweed species were chosen as part of an experiment designed to investigate patterns of local adaptation (e.g. monarch population $\mathrm{x}$ milkweed species interactions) to host plants across the monarch's global range (Freedman et al., in prep). As such, only two of the milkweed species included here are commonly encountered by monarchs in North America. We grew Asclepias syriaca as our eastern North American host plant, A. fascicularis as our western North American host plant, A. curassavica (Guam: Mariana Islands), Gomphocarpus physocarpus (Maui: Hawaiian Islands), and G. fruticosus (Queensland: Australia). For the purposes of analysis, we combine results from the two Gomphocarpus species, as they are close relatives that potentially hybridize in their native and introduced ranges [24,25], and are virtually indistinguishable outside of minor differences in fruit and flower morphology [25]. For a summary of monarch populations, number of maternal families, and host plant species tested, see Table S1.

We reared approximately 225 caterpillars from 25 maternal families in mesh bags on live host plants (i.e. not clipped leaves) grown from seed in a greenhouse. We then collected adult butterflies that eclosed from each host plant and recorded their wing morphology in the same way as described above.

To evaluate these data, we used a linear mixed model with wing area or elongation as our response variables. We treated maternal family as well as greenhouse and greenhouse block as random intercept terms, with fixed effects for monarch population and host plant species. 
Comparisons within factor levels (i.e. pairwise differences between host plants and monarch populations) were made using Tukey HSD tests.

\section{Results}

As in previously published studies, male monarchs had forewings that were $1.8 \%$ larger than females $(\mathrm{t}=$ $6.41, \mathrm{p}<0.001$ ) (Table 2), corresponding to an absolute difference of $15.9 \pm 2.5 \mathrm{~mm}^{2}$. Overwintering butterflies were $4.4 \%$ larger than non-overwintering butterflies $(\mathrm{t}=$ 5.06, $\mathrm{p}<0.001$ ), and there was a significant interaction between overwintering status and presence in eastern versus western North America driven by discrepancies in the size of overwintering individuals $(\mathrm{t}=2.24, \mathrm{p}=$ 0.029 , Figure 3). Wing area increased with latitude $(t=$ $2.80, p=0.008$, Figure 4), such that monarchs collected from the northernmost extent of the migratory range were approximately $6 \%$ larger than monarchs from nonoverwintering areas in the southernmost areas of the monarch's range. Butterflies also become larger through time $(\mathrm{t}=2.23, \mathrm{p}=0.026)$, with wing area increasing by approximately $4.9 \%$ (an absolute difference of $43.5 \pm 19.5$ $\mathrm{mm}^{2}$ ) between 1878-2017 (Figure 5a).



Figure 3. Overwintering individuals are significantly larger than non-overwintering individuals across the dataset. There was a significant interaction between overwintering status and membership in eastern versus western North America, such that summer breeding butterflies are approximately the same in both locations, but butterflies overwintering in Mexico are larger than butterflies overwintering in California.

Table 2. ANOVA results for models assessing (a) forewing area and (b) elongation. Predictors with $p<0.1$ are shown in bold, with asterisks corresponding to levels of significance. Results were calculated using type III sums of squares in the car package [55].

(a). Response variable: Forewing area

\begin{tabular}{|c|c|c|c|}
\hline Predictor & DF & $x^{2}$ & $\mathbf{p}$ \\
\hline Sex & 1 & 41.02 & $<0.001 * \star \star *$ \\
\hline Year & 1 & 4.95 & $0.026 *$ \\
\hline Overwintering status & 1 & 25.65 & $<0.001 * * *$ \\
\hline East vs. west & 1 & 3.60 & 0.058 \\
\hline Latitude & 1 & 7.82 & $0.005^{\star \star}$ \\
\hline Daylength index & 1 & 3.63 & 0.057 \\
\hline Overwintering status * East vs. west & 1 & 5.01 & $0.025^{*}$ \\
\hline \multicolumn{4}{|c|}{ (b). Response variable: Forewing elongation } \\
\hline Predictor & DF & $x^{2}$ & $\mathbf{p}$ \\
\hline Sex & 1 & 3.68 & 0.055 \\
\hline Year & 1 & 1.83 & 0.176 \\
\hline Overwintering status & 1 & 1.60 & 0.205 \\
\hline East vs. west & 1 & 1.43 & 0.232 \\
\hline Latitude & 1 & 5.32 & $<0.021 *$ \\
\hline Daylength index & 1 & 3.47 & 0.062 \\
\hline Overwintering status * East vs. west & 1 & 0.97 & 0.325 \\
\hline
\end{tabular}


Table 3. ANOVA results for experiment assessing the contribution of milkweed host plant, sex, and monarch population for (a) forewing size and (b) forewing roundness. Milkweed species contributes strongly to variation in forewing size but not roundness.

(a). Response variable: Forewing area

\begin{tabular}{llll}
\hline Predictor & DF & X2 & p \\
Milkweed species & $\mathbf{3}$ & $\mathbf{1 7 . 7 6}$ & $<.001^{\star \star *}$ \\
Sex & 1 & 2.95 & 0.086 \\
Monarch population & 3 & 5.10 & 0.164 \\
\hline (b). Response variable: Forewing elongation & & p & 0.155 \\
\hline Predictor & DF & 5.23 & $\mathbf{0 . 0 2 2 ^ { * }}$ \\
Milkweed species & 3 & 5.25 & 0.524 \\
Sex & 1 & 2.24 & \\
\hline
\end{tabular}

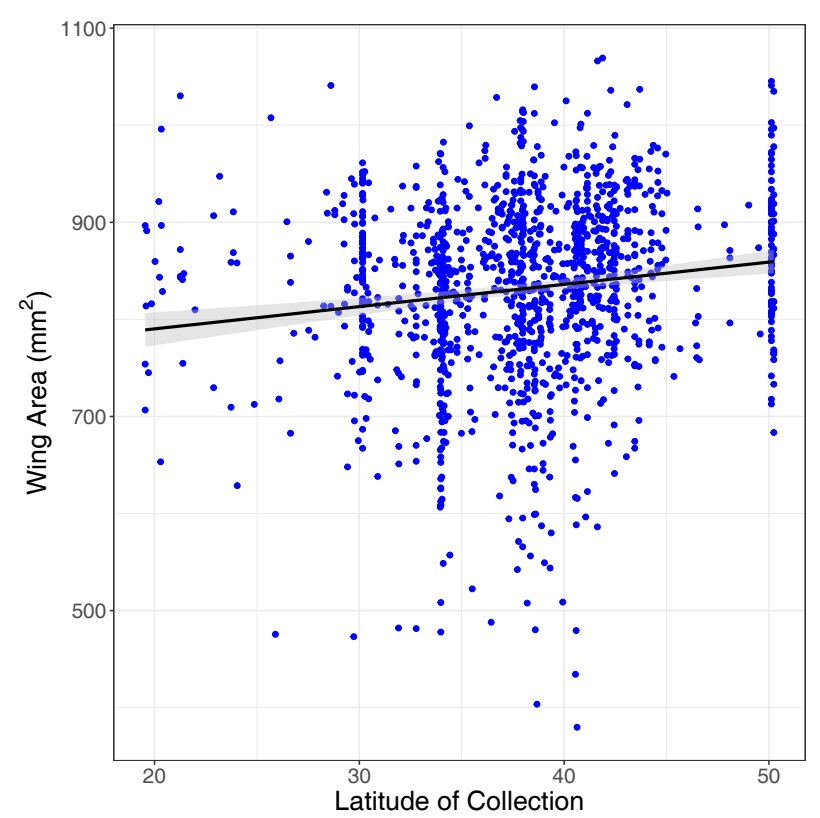

Figure 4. Monarch forewing area increases significantly with latitude of collection. Points shown here do not include overwintering individuals. Shaded areas around trend lines correspond to $95 \%$ confidence intervals.

In contrast to wing area, the only significant predictor for wing elongation was latitude, with butterflies collected at higher latitudes having significantly more elongated wings $(t=2.16, p=0.035)$ (Table 2). Sex was a marginally significant predictor of wing elongation, such that males had slightly more elongated wings than females $(t=1.92, p$ $=0.055$ ). Butterflies collected during shorter daylengths had slightly more elongated wings $(\mathrm{t}=1.86, \mathrm{p}=0.063)$ (Table 2$)$.

When we reanalyzed the data in Flockhart et al. (2017) [17], we found an increase of $1.9 \%$ for wing area between 1974-2014 $(\mathrm{t}=2.06, \mathrm{p}=0.040)$ and $1.4 \%$ for wing length $(t=2.74, p=0.006)$ (Figure $5 b)$, even after accounting for inferred migration distance. We found the same pattern when we restricted our main dataset to the same time period and included only non-overwintering butterflies, with a 5.5\% increase through time for wing area between 1974-2014 $(\mathrm{t}=2.26, \mathrm{p}=0.026)$ and a $2.9 \%$ increase for wing length $(t=2.18, p=0.032)$ (Figure $5 c$ ).

We found strong effects of larval host plant identity on adult wing size (Table 3). Specifically, monarchs had the largest forewings on Asclepias syriaca compared to other tested species, with forewings that were $8.7 \%$ larger than monarchs reared on A. fascicularis $(\mathrm{z}=3.943, \mathrm{p}<0.001)$, 6.2\% larger than monarchs reared on $A$. curassavica $(\mathrm{z}=$ $3.090, \mathrm{p}=0.010)$, and $4.1 \%$ larger than monarchs reared on Gomphocarpus spp. $(\mathrm{z}=2.374, \mathrm{p}=0.080)$ (Figure 6). By contrast, there were no host plant effects on wing elongation (Table 3). Wing area and body mass were highly correlated (Figure S3).

\section{Discussion}

Migratory North American monarch butterflies show substantial variation in their wing morphology, although the factors contributing to this variation remain relatively poorly characterized. Here, we use a large dataset and show that sex, overwintering status, and latitude of collection all explain a substantial proportion of the observed variation in monarch forewing size. Additionally, we find a significant increase in the size of North American monarch forewings through time, a pattern that has not been observed before and that was corroborated by re-analysis of the data from Flockhart et al. (2017) [17]. Finally, we show that larval host plant identity can have strong impacts on monarch wing morphology, with certain milkweed species such as A. syriaca supporting especially large butterflies. 

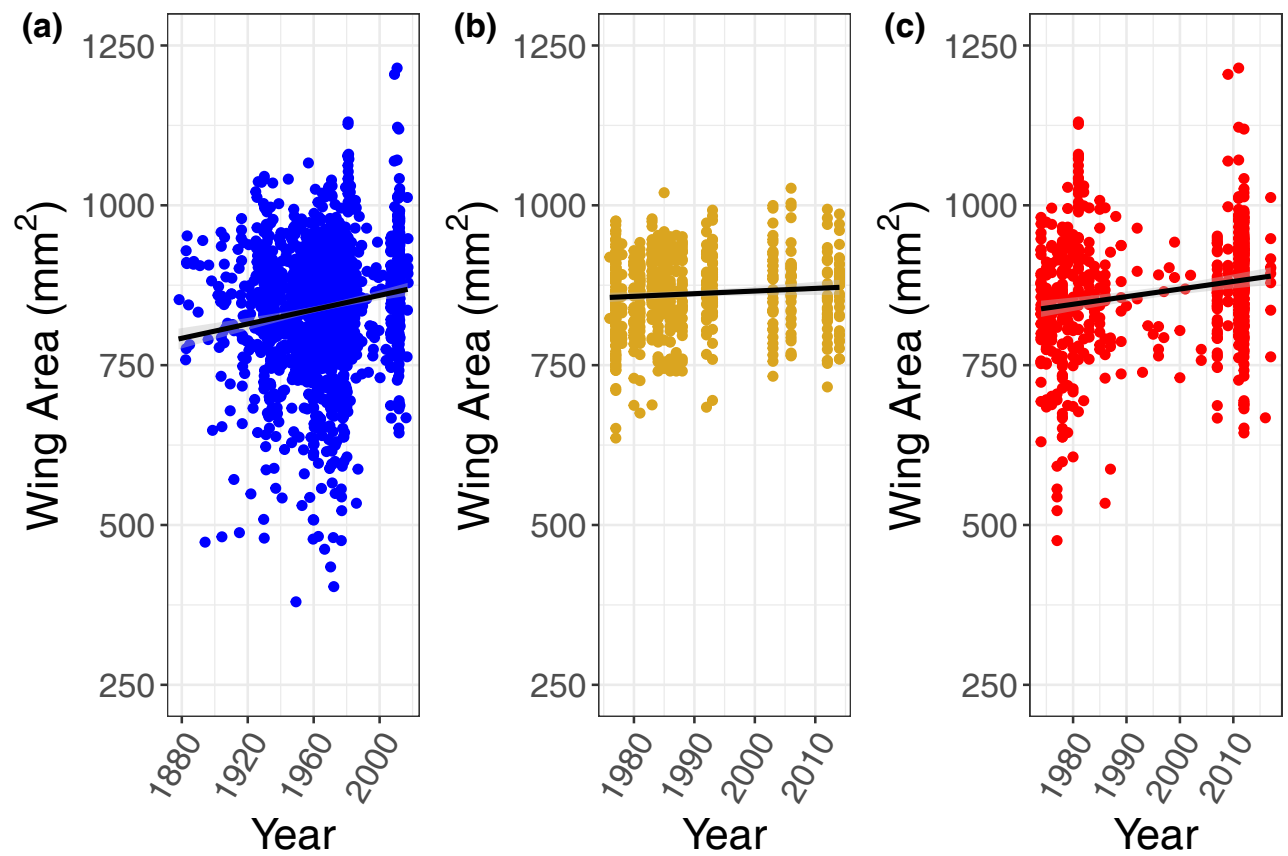

Figure 5. (a) Our dataset, including all observations, shows a significant increase through time in wing area. (b) The Flockhart data using Mexican overwintering individuals also shows a significant increase in wing area through time. (c). Our data from summer breeding butterflies, restricted to the same time interval as the Flockhart data, also shows a significant increase through time. Points shown in (a) and (c) do not include overwintering individuals. Shaded areas around trend lines correspond to $95 \%$ confidence intervals.

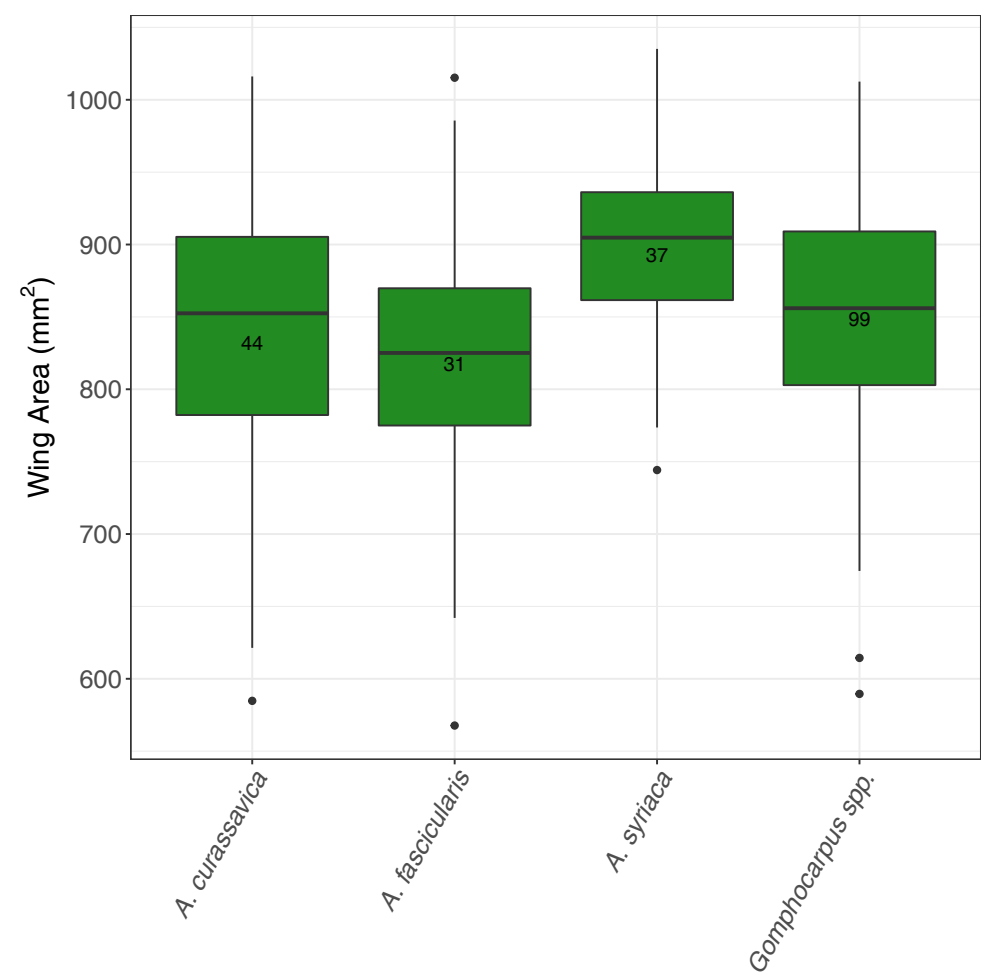

Figure 6. Monarch forewing area varies substantially based on larval host plant. Adults reared on $\mathrm{A}$. syriaca were significantly larger than adults reared on $A$. curassavica $(p=0.009)$ and $A$. fascicularis $(p<0.001)$, and marginally larger than adults reared on Gomphocarpus $(p=$ 0.076). Numbers inside bars correspond to pooled sample sizes for any particular host species. Gomphocarpus spp. includes both Gomphocarpus fruticosus and G. physocarpus. 
The most consistent source of variation in our dataset was sex, with male butterflies having forewings that were approximately $1.8 \%$ larger and marginally more elongated than female butterflies. Sexual dimorphism in forewing size has been documented in numerous studies of monarchs [9] and has generally been interpreted as the result of sexual selection favoring larger males, possibly due to increased ability to overcome female resistance to mating, female preference, or sperm competition $[26,27]$. Larger size in males could also be related to monarch mating behavior, which involves a lengthy copulation and post-nuptial flight period of up to 16 hours in which males fly with females attached [28]. Male forewings were slightly more elongated than female forewings, a pattern that has not been shown before. This result seems to be partly driven by the inherent correlation between forewing area and forewing roundness $\left(R^{2}=0.25\right.$, Figure $\left.S 2\right)$, whereby larger forewings also tend to be more elongated.

One possible implication of the discrepancy in size between males and females is differing migration capabilities based on sex. This discrepancy in wing size could contribute to differing migration success between sexes, with males potentially better suited for longdistance migration. Although neither Yang et al. (2016) [16] nor Flockhart et al. (2017) [17] found sex-based differences in inferred migration distance, the increasingly malebiased sex ratio at overwintering sites in Mexico could potentially reflect sex-based differences in migration ability and/or distance [26]. However, this runs counter to tagging data from Steffy (2015) [29], which indicated that female monarchs have higher success in reaching Mexico than males.

The next largest source of variation was overwintering status, with monarchs collected from overwintering sites being significantly larger than those away from overwintering sites. This result is consistent with previous findings and supports the idea that longdistance migration to overwintering locations may act as a selective episode on wing morphology, such that only the most capable migrants eventually reach these sites $[16,17]$. An alternative explanation for this finding is that summer breeding butterflies that develop under warmer temperatures and longer photoperiod might be smaller as a result of phenotypic plasticity. Temperature and photoperiod effects on adult size and wing morphology have been documented in monarchs [19,31]. However, the daylength index was only a modest predictor of wing size in our dataset (Table 2) and in the opposite direction predicted, with forewing size being slightly larger for butterflies collected during longer days, suggesting that photoperiod per se does not strongly drive patterns of wing morphological variation. Likewise, Flockhart et al. (2017) [17] did not find strong support for a statistical model that included temperature in the natal range as a predictor of adult wing morphology.

We found a significant interaction between overwintering status and membership in the eastern versus western North America population of butterflies. This pattern was driven by larger overwintering butterflies in eastern compared to western North America; by contrast, there were no apparent differences in wing morphology between eastern versus western summer breeding butterflies (Figure 3). Furthermore, when monarchs from eastern North America and California were reared together on the same host plants and in the same environment, we did not find any differences between butterflies from east versus west $(z=0.21, p>0.8)$. This result makes sense in light of the recent insight that all North American monarchs form a randomly mating panmictic population, with enough exchange of migrants at Mexican overwintering sites to collapse any pattern of genetic differentiation [32,33]. These results suggest that it is the nearly ten-fold difference in average migration distance between eastern and western overwintering monarchs that drives differences in wing area [8], rather than any genetic differentiation between populations. Note, however, that all western overwintering butterflies included in our study were collected in California, even though some portion of western summer breeders apparently migrate to Mexico in the autumn [34].

We also found a significant effect of latitude of collection for both wing area and elongation. This result contrasts with those of Li et al. (2016) [11], who did not find evidence for a latitudinal cline in wing morphology and instead suggested that migratory status rather than latitude drives patterns of monarch wing morphology. Latitudinal clines in body size related traits are ubiquitous across both endo- and ectotherms [35,36], with size typically increasing with latitude as we saw in our data. However, it is important to note that latitude of collection for adult monarchs may be a poor proxy for the latitude at which larvae developed, which is when adult wing morphology is determined. This may be especially true for butterflies collected during the spring and fall migration periods, as individuals collected during these intervals are more likely to have originated from distant locations.

There are multiple possible reasons for the latitudinal cline in wing morphology. The first and most parsimonious explanation is that migration acts as a selective filter during the spring re-migration away from overwintering sites, such that only the most capable migrants reach more northerly latitudes. A second hypothesis relates to 
development rate and temperature, whereby caterpillars that develop under cooler temperatures such as those that might be encountered at higher latitudes are more likely to grow large $[35,36]$; however, we again note that Flockhart et al. (2017) [17] found no relationship between temperature in the inferred natal range and wing area for Mexican overwintering butterflies. A final possible explanation is that host plants that occur further north in the monarch's North American range support especially large butterflies, an explanation that we discuss later.

The final predictor that explained a substantial portion of the variation in North American monarch wing morphology was the year of collection, with butterflies increasing significantly in size over our sampling window. This pattern is intriguing and has not been documented for monarch butterflies before. This result was further corroborated by reanalyzing the data from Flockhart et al.'s (2017) [17] Mexican overwintering butterflies, which also show a significant increase in wing size through time. While the Flockhart paper chose to include year as a random effect to account for stochastic year-to-year variability (e.g. annual differences in climate suitability of breeding grounds), our overall dataset includes observations from 119 unique years, which should integrate over the impacts of any stochastic year-to-year variation. Both datasets indicate a significant size increase through time, although the magnitude of this increase seems to be greater in the summer breeding $\left(1.18 \pm 0.54 \mathrm{~mm}^{2} /\right.$ year $)$ compared to overwintering butterflies $\left(0.44 \pm 0.21 \mathrm{~mm}^{2} /\right.$ year). In contrast, neither our data nor the Flockhart data suggest a change in forewing elongation through time. Combining our results with those of Flockhart et al. provides compelling evidence that the observed increase in size through time is not simply an artifact of small sample sizes or stochastic year-to-year variability.

In assessing the result of increased size through time, it is important to keep two things in mind: the first is that the absolute change in wing area over the sampling window is still relatively modest, corresponding to an overall increase of approximately $4.9 \%\left(43.6 \mathrm{~mm}^{2}\right)$ over 140 years. By comparison, the difference in any given year between overwintering and summer-breeding butterflies is 4.4\% (38.8 $\mathrm{mm}^{2}$ ), and the average size difference between a monarch collected in southern Texas versus southern Manitoba is $4.7 \%\left(41.1 \mathrm{~mm}^{2}\right)$. More functional studies linking wing morphology and flight performance [e.g. 37,38] are needed to fully understand how biologically meaningful the increase in wing area may be. Second, there are a number of nonmutually exclusive explanations for the observed pattern of increased forewing size through time, and we do not claim to understand exactly why we see this pattern.
One possible explanation is that there is ongoing directional selection for increased forewing size, whereby monarchs that are larger have a relative fitness advantage and are therefore shifting the overall mean phenotypic value towards larger wing sizes. However, monarchs have been migratory over large spatial scales within North America for an estimated 20,000 years (and probably longer) [39,40], so it is not immediately clear why they would not have already been near their optimum wing morphological phenotype. Another possible explanation is that the increased size through time reflects a change in the natal origins of monarchs over the sampling window, such that monarchs reaching the overwintering grounds and subsequently recolonizing North America are increasingly derived from more distant locations. Flockhart et al. (2017b) [41] found no evidence for the hypothesis that natal origins changed during the time period between 1974-2014. However, other studies have suggested that the monarch's breeding range may be shifting northward [42], and there is evidence that the pace of the monarch's southward migration is becoming faster, potentially as a consequence of a northward range shift [43].

Yet another possible reason for the increase in size through time is that the assemblage of milkweed host plants in North America has changed over the sampling window. While the absolute abundance of milkweeds has likely decreased precipitously throughout part of the monarch's breeding range in the past two to three decades [44,45], less is known about the relative abundance of individual milkweed species. If species such as $A$. syriaca that support especially large adult butterflies have become relatively more frequent over the sampling window, this could yield an apparent increase in size for the overall population. This hypothesis is supported by a recent analysis of herbarium records for ten common North American milkweeds, including more than 3000 records of A. syriaca from 1900-present [46]. While overall milkweed abundance declined over the sampling window (especially since 1940), the relative abundance of $A$. syriaca increased during this time.

Lastly, the observed increase in monarch size may also be attributable to climate change, which could influence monarch forewing size in at least two ways. First, climate change may make areas of the southern and central U.S. unsuitable for summer breeding monarchs due to thermal constraints on caterpillar development [42,47-49], thereby forcing monarchs further north during the summer and leading to fall migrants that must migrate longer distances. Second, climate change may be expanding the northern range edge of some North American milkweeds such as $A$. 
syriaca and by extension expanding the northern extent of the monarch's North American range [42]. However, this latter hypothesis seems unlikely to generate a discernible change in monarch wing morphology given the relative rate at which milkweed species' ranges may be expanding compared to the overall distance covered by migratory monarchs. Comparison of contemporary records of Asclepias with those listed in Woodson (1954) [50] and those compiled by Boyle et al. [46] would be useful to understand how climate change may be shifting the northern range limit of certain milkweed species.

Our rearing experiment showed that larval host plant has a strong influence on adult wing morphology. This pattern has been documented in one other study [18], which showed that adult forewing length varies by up to $4.5 \mathrm{~mm}$ on co-occurring Asclepias species in Iowa. A difference of this magnitude is comparable or even larger than that observed between migratory and non-migratory populations of butterflies [8,11], suggesting a strong environmental component for variation in wing size. Host plant effects may also explain why there is a slight excess of small butterflies in the overall distribution of forewing areas for wild caught butterflies (Figure 3, Figure S2), as these individuals could reflect butterflies that developed on poor-quality milkweed species or caterpillars that pupated prematurely, potentially due to food limitation [51]. By contrast, we saw no impact of host plant identity on wing elongation, suggesting a smaller contribution of environmental influences for monarch wing shape measurements. The sequential nature of the monarch's spring re-migration brings it into contact with distinct assemblages of milkweed species as it moves north [42,52], and understanding how each of these species influences adult size would be a useful avenue for future research into wing morphological variation. Because our host plant experiment only included two North American milkweed species, evaluating the influence of milkweed species on migratory wing morphology will require more thorough investigation.

Our paper highlights the need for more careful consideration of the full range of factors that may contribute to variation in monarch butterfly wing morphology as well as the utility of using large datasets to test hypotheses. For example, our results contrast with previous published works suggesting that there are no latitudinal clines in North America [11] and that there are inherent size differences between eastern and western North American butterflies [8]. Future studies that focus on naturally occurring patterns of variation in monarch wing morphology should be mindful of the myriad environmental influences (natal host plant species, developmental photoperiod and diapause status, developmental temperature) that may contribute to this variation. Finally, we highlight the tremendous utility of using museum collections [53,54], without which we could not have detected changes through time or achieved such a wide geographical range of samples.

Acknowledgments: We thank Amanda Pierce, Yiwen Li, Louie Yang, Myron Zalucki, and David M. for providing images. We thank Asia Jones, Jacob Dehkordi, and Jessica Liaudat for assistance with wing measurements. Assistance with the host plant experiment was provided by Asia Jones, Anika Hamilton, Moria Robinson, Nick Pardikes, Sharon Strauss, Kyle Christie, Jenna Yonenanga, and Jessica Aguilar. Sharon Strauss, Santiago Ramirez, Louie Yang, and Myron Zalucki provided helpful feedback on ideas presented in this paper. We also thank numerous museum collections and their curators: Courtney Richenbacher and Dave Grimaldi (AMNH), Jim Boone (Bishop Museum), Lynn Kimsey and Steve Hayden (Bohart Museum), Jere Schweikert (Cal Academy), Jason Dombroskie (Cornell University), Peter Oboyski (Essig Museum), Rachel Hawkins (Harvard MCZ), Weiping Xie (LACMNH), Andrei Sourakov (McGuire Center), Lawrence Gall (Peabody Museum), Doug Yanega (UC Riverside), and Bob Robbins (Smithsonian). Funding for M.F. was provided by an NSF Graduate Research Fellowship as well as a Rosemary Grant Award from the Society for the Study of Evolution and an Explorer Grant from the National Geographic Society. H.D. was funded by a UC Davis Dickson Emeritus Grant. All data and code can be found at https://github.com/micahfreedman/Freedmanand-Dingle-2018.

\section{References}

[1] Herman, W.S., Hormonally mediated events in adult monarch butterflies. In Rankin, M.A. (ed.), Migration: Mechanisms and Adaptive Significance, Contrib. Mar. Sci. 1985, 27, 799-815.

[2] Dingle, H., Migration: The Biology of Life on the Move, $2^{\text {nd }}$ edition, Oxford University Press, Oxford, United Kingdom, 2014.

[3] Assumpção L. et al., The use of morphometric analysis to predict the swimming efficiency of two Neotropical long-distance migratory species in fish passage, Neotrop. Ichthyol., 2012, 10, 797-804.

[4] Chapman, B.B. et al., Shape up or ship out: migratory behaviour predicts morphology across spatial scale in a freshwater fish, J. Anim. Ecol., 2015, 84, 1187-1193.

[5] Winkler, H. and Leisler, B., To be a migrant: Ecomorphological burdens and chances. In Greenberg, R. \& Marra, P. (eds.). Birds of Two Worlds: the Ecology and Evolution of Migration, pp. 79-86. Johns Hopkins University Press, Baltimore, MD, 2005. 
[6] Bowlin, M.S. and Wikelski, M., Pointed wings, low wing loading and calm air reduce migratory flight costs in songbirds, PLoS One, 2008, 3, e2154.

[7] Dingle, H. et al., Variation in body size and flight performance in milkweed bugs (Oncopeltus), Evolution 1980, 34, 371-385.

[8] Altizer, S. and Davis, A.K., Populations of Monarch butterflies with different migratory behaviors show divergence in wing morphology, Evolution, 2010, 64, 1018-1028.

[9] Beall, G. and Williams, C.B., Geographical variation in the wing length of Danaus plexippus (Lep. Rhopalocera), Proceedings of the Royal Entomological Society of London 1945, 20, 65-76.

[10] Dockx, C., Directional and stabilizing selection on wing size and shape in migrant and resident monarch butterflies, Danaus plexippus (L.), in Cuba, Biol. J. Linn. Soc., 2007, 92, 605-616.

[11] Li, Y. et al., Variation in forewing size linked to migratory status in monarch butterflies, Animal Migration, 2016, 3, 27-34.

[12] Gibo, D.L. and McCurdy, J.A., Lipid accumulation by migrating monarch butterflies (Danaus plexippus L.)., Can. J. Zool., 1973, 71, 76-82.

[13] Davis, A.K., Wing color of monarch butterflies (Danaus plexippus) in eastern North America across life stages: Migrants Are "redder" than breeding and overwintering stages, Psyche, 2009, Article ID 705780.

[14] Hanley, D. et al., Forewing pigmentation predicts migration distance in wild-caught migratory monarch butterflies, Behav. Ecol., 2013, 24, 1108-1113.

[15] Satterfield, D.A. and Davis, A.K., Variation in wing characteristics of monarch butterflies during migration: Earlier migrants have redder and more elongated wings, Animal Migration, 2014, 2, 1-7.

[16] Yang, L.H. et al., Intra-population variation in the natal origins and wing morphology of overwintering western monarch butterflies Danaus plexippus, Ecography, 2016, 39, 998-1007.

[17] Flockhart, D.T.T. et al., Migration distance as a selective episode for wing morphology in a migratory insect., Mov Ecol, 2017, 5:7.

[18] Pocius, V.M. et al., Milkweed matters: Monarch butterfly (Lepidoptera: Nymphalidae) survival and development on nine Midwestern milkweed species, Environ. Entomol., 2017, 46, 1098-1105.

[19] Freedman, M.G. et al., Non-migratory monarch butterflies, Danaus plexippus (L.), retain developmental plasticity and a navigational mechanism associated with migration, Biol. J. Linn. Soc., 2018, 123, 265-278.

[20] Schneider, C.A. et al., NIH Image to Image): 25 years of image analysis, Nat. Methods, 2012, 9, 671-675.

[21] Kahle, D. and Wickham, H., ggmap: Spatial visualization with ggplot2. The R Journal, 2013, 5, 144-161.

[22] Bates, D. et al., Fitting linear mixed-effects models using Ime4, Journal of Statistical Software, 2015, 67: 1-48.

[23] R Core Team. R: A language and environment for statistical computing, 2013, R Foundation for Statistical Computing, Vienna, Austria.

[24] Ward M., Johnson S.D., Zalucki M.P., Modes of reproduction in three invasive milkweeds are consistent with Baker's Rule, Biol. Invasions, 2012, 14, 1237-1250

[25] Goyder D.J., Nicholas A., A revision of Gomphocarpus R. Br. (Apocynaceae: Asclepiadeae), Kew Bull., 2001, 56, 769-836.
[26] Davis, A.K. et al., Wing color predicts future mating success in male monarch butterflies, Ann. Entomol. Soc. Am., 2007, 100, 339-344.

[27] Solensky, M.J. and Oberhauser, K.S. Sperm precedence in monarch butterflies (Danaus plexippus), Behav. Ecol., 2009, 20, 328-334.

[28] Hill H.F., Wenner A.M., Wells P.H., Reproductive behavior in an overwintering aggregation of monarch butterflies, Am. Midl. Nat., 1976, 95, 10-19.

[29] Steffy G., Trends observed in fall migrant monarch butterflies (Lepidoptera: Nymphalidae) east of the Appalachian Mountains at an inland stopover in southern Pennsylvania over an eighteen year period, Ann. Entomol. Soc. Am., 2015, 108, 718-728.

[30] Davis, A.K. and Rendón-Salinas, E., Are female monarch butterflies declining in eastern North America? Evidence of a 30-year change in sex ratios at Mexican overwintering sites, Biol. Lett., 2015, 6, 45-47.

[31] York, H.A. and Oberhauser, K.S, Effects of duration and timing of heat stress on monarch butterfly (Danaus plexippus) (Lepidoptera: Nymphalidae) development, J. Kans. Entomol. Soc., 2002, 75, 290-298.

[32] Lyons, J.I. et al., Lack of genetic differentiation between monarch butterflies with divergent migration destinations, Mol. Ecol., 2002, 21, 3433-3444.

[33] Pierce, A.A. et al., Serial founder effects and genetic differentiation during worldwide range expansion of monarch butterflies, Proc. Roy. Soc. B, 2014, 281, 20142230.

[34] Dingle, H. et al., Distribution of the monarch butterfly, Danaus plexippus (L.)(Lepidoptera: Nymphalidae), in western North America, Biol. J. Linn. Soc., 2005, 85, 491-500.

[35] Atkinson, D., Temperature and organism size: a biological law for ectotherms? Adv. Ecol. Res., 1994, 25, 1-58.

[36] Kingsolver, J.G. and Huey, R.B., Size, temperature, and fitness: three rules. - Evol. Ecol. Res., 2008, 10, 251-268.

[37] Bradley, C.A. and Altizer, S., Parasites hinder monarch butterfly flight: implications for disease spread in migratory hosts, Ecol. Lett., 2005, 8, 290-300.

[38] Davis, A.K. et al., The redder the better: wing color predicts flight performance in monarch butterflies, PLoS One, 2012, 7, e41323.

[39] Zhan, S. et al., The genetics of monarch butterfly migration and warning colouration, Nature, 2014, 514, 317-321.

[40] Pfeiler, E. et al., Population genetics of overwintering monarch butterflies, Danaus plexippus (Linnaeus), from central Mexico inferred from mitochondrial DNA and microsatellite markers, J. Hered. 2017, 108, 163-175.

[41] Flockhart, D.T.T. et al., Regional climate on the breeding grounds predicts variation in the natal origin of monarch butterflies overwintering in Mexico over 38 years, Glob. Chang. Biol., 2017, 23, 2565-2576.

[42] Batalden R.V., Oberhauser K., Peterson A.T., Ecological niches in sequential generations of eastern North American monarch butterflies (Lepidoptera: Danaidae): The ecology of migration and likely climate change implications, Environ. Entomol., 2007, 36, 1365-1373.

[43] Howard, E., and Davis, A.K. 2015. Tracking the fall migration of eastern monarchs with Journey North roost sightings: New findings about the pace of fall migration, pp. 207-214. In K. Oberhauser, S. Altizer and K. Nail (eds.), Monarchs in a 
changing world: Biology and conservation of an iconic insect. Cornell University Press, Ithaca, NY.

[44] Pleasants, J.M. and Oberhauser, K.S., Milkweed loss in agricultural fields because of herbicide use: effect on the monarch butterfly population, Insect Conserv. Divers., 2013, 6, 135-144.

[45] Zaya, D.N. et al., Long-term trends in Midwestern milkweed abundances and their relevance to monarch butterfly declines, Bioscience, 2017, 67, 343-356.

[46] Boyle, J.H., Dalgleish, H.J. and Puzey, J. Monarch butterfly and milkweed declines substantially predate the use of genetically modified crops, BioRxiv preprint, 2018, https://doi. org/10.1101/378299.

[47] Zalucki M.P., Temperature and rate of development in Danaus plexippus L. and D. chrysippus L. (Lepidoptera: Nymphalidae), Aust. J. Entomol., 1982, 21, 241-246

[48] Malcolm, S.B. et al., Monarch butterfly voltinism: Effects of temperature constraints at different latitudes, Oikos, 1987, 49, 77-82.

[49] Zalucki M.P. and Rochester W.A., Spatial and temporal population dynamics of monarchs down-under: lessons for North America. In: The Monarch Butterfly: Biology and
Conservation (eds K Oberhauser \& M Solensky), pp. 219-228. Cornell University Press, Ithaca, USA, 2004.

[50] Woodson, R.E., The North American species of Asclepias L., Ann. Mo. Bot. Gard., 1954, 41, 1-211.

[51] Johnson, H. et al., Does skipping a meal matter to a butterfly's appearance? Effects of larval food stress on wing morphology and color in monarch butterflies, PLoS One, 2014, 9, e93492.

[52] Flockhart D.T.T., et. al., Tracking multi-generational colonization of the breeding grounds by monarch butterflies in eastern North America, Proc. Roy. Soc. B., 2013, 280, 20131087.

[53] Suarez, A. and Tsutsui, N.D. The value of museum collections for research and society. Bioscience, 2005, 54, 66-74.

[54] Holmes, M.W. et al., Natural history collections as windows on evolutionary processes, Mol. Ecol., 2016, 25: 864-881.

[55] Fox, J. \& S. Weisberg, An 'R' companion to applied regression, $2^{\text {nd }}$ edition. Sage Publishing, Thousand Oaks, CA, 2011.

[Supplemental Material: The online version of this article [(DOI: 10.1515/ami-2018-0003) offers supplementary material. 